agreement test for different raters in systematic reviews 3 . How to from evidence to recommendations in guidelines 4. Exploration of the application of GRADE in clinical stage (e.g. whether the guideline covers prevention, screening, assessment, treatment, rehabilitation or monitoring); Exploration of the application of GRADE in different areas such as public health, health policy and system. 5. The establishment and development of GRADE centre such as the Chinese GRADE centre and Spanish GRADE centre and how to promote the implementation of GRADE.

Conclusion As a new paradigm, the GRADE approach provides a comprehensive, explicit, and transparent methodology for grading the quality of evidence and strength of recommendations about the management of patients, however, GRADE is not a perfect system and still need to develop itself and been disseminated widely.

\section{P044 QUALITY ASSESSMENT OF TRADITIONAL CHINESE CLINICAL GUIDELINES: 2003-2012}

${ }^{1,2} \mathrm{Y}$ Chen, ${ }^{1,2} \mathrm{~L}$ Yao, ${ }^{1,2} \mathrm{Q}$ Wang, ${ }^{1,2} \mathrm{D}$ Wei, ${ }^{1,2} \mathrm{X}$ Wang, ${ }^{1,2} \mathrm{~K}$ Yang. ${ }^{1}$ Evidence-Based Medicine Center of Lanzhou University, Lanzhou, China; ${ }^{2}$ Chinese GRADE Center, Lanzhou, China

\section{0:1136/bmjqs-2013-002293.138}

Background Little is known about quality and quantity of traditional Chinese clinical guidelines. We aim to systematically review all of traditional Chinese clinical guidelines.

Methods We searched CNKI (China National Knowledge Infrastructure/Chinese Academic Journals full text Database), VIP (a fulltext database of China), WANFANG (a fulltext database of China) and CBM (China Biomedicine Database Disc). Two groups of review authors independently applied inclusion criteria, assessed trial quality, and extracted data.

Results We identified 75 traditional Chinese clinical guidelines from 2003 to 2012, and only 11(15\%) were claimed that an evidence based approached were used in the process of development. From the assessment with the Appraisal of Guidelines for Research and Evaluation II (AGREE II), the mean scores were low for the domains 'clarity of presentation' (28\%), 'scope and purpose' $(15 \%)$ and 'editorial independence' $12 \%$; and very low for the other domains ('stakeholder involvement' $8 \%$, 'rigour of development' 5\% and 'applicability' 3\%). AGREE II mean scores of traditional Chinese clinical guidelines lower than Chinese clinical practice guidelines and the world average.

Conclusions Traditional Chinese clinical guidelines received lower scores, which indicate a relatively poor quality of the guidelines. However, there was some increase over time. Meanwhile, given the characteristics of Traditional Chinese medicine, CONSORT group has been developing CONSORT for TCM and for Acupuncture, we plan to develop AGREE TCM to be used to inform the development, appraisal and reporting of evidence-informed traditional Chinese clinical guidelines.

\section{P049 EVIDENCE DISTRIBUTION OF A COMPREHENSIVE MUSCULO-SKELETAL GUIDELINE}

${ }^{1} \mathrm{C}$ Wolfkiel, ${ }^{1} \mathrm{~J}$ Ording, ${ }^{2} \mathrm{M}$ Thiese, ${ }^{3} \mathrm{M}$ Stanhope, ${ }^{4} \mathrm{~J}$ Harris, ${ }^{5} \mathrm{~K}$ Hegmann. ${ }^{1}$ American College of Occupational and Environmental Medicine, Elk Grove Village, USA; ${ }^{2}$ University of Utah-Rocky Mountain Center on Occupational and Environmental Health, Salt Lake City, USA; ${ }^{3}$ Indico, Ltd, Melbourne, Victoria, Australia; ${ }^{4}$ The Permanente Medical Group, San Rafael, USA; ${ }^{5}$ University of Utah-Rocky Mountain Center on Occupational and Environmental Health Salt Lake City, USA

10:1136/bmjqs-2013-002293.139
Background Occupational Medicine focuses on return to functionality and work, however the overwhelming majority of injuries are musculoskeletal and require very specific clinical situation evidence review. Body part areas addressed include spine, shoulder, elbow, hand/wrist/forearm, hip/groin, knee and ankle/foot. Interventions assessed include diagnostic, therapeutic and medical therapies.

Objectives To assess the theoretical evidence distribution of a comprehensive musculoskeletal guideline and its potential application in practice. Methods: Evidence ratings (A,B,C) were determined by expert data extraction from over 5,000 randomised controlled trials (RCT), non-RCT evidence was designated as insufficient (I). RCT evidence ratings were quantified on an 11 point scale that assessed appropriateness, biases and effectiveness. High quality evidence was defined as $8.0-11$ points, moderate 4.0-7.5, and low $<4.0$ points. A level evidence (Strong) was defined as 2 or more high-quality RCTs, B (Moderate) 1 high-quality or multiple moderate-quality, C (Limited) at least one study of moderate-quality. Low quality, observational or conflicting evidence was designated as Insufficient (I). A similar profile was used for diagnostic evidence recommendations. Evidence ratings were verified by independent writing panels.

Results Only 0\% of 2500 recommendations were supported by a Limited (C) or better evidence base. When adjusting for frequency of occurrence from a claims data base, and cost was estimated in only $14 \%$ of costs were associated with quality RCT studies.

Conclusions These data suggest that the majority of musculoskeletal clinical decisions do not have a sufficient evidence base for rules-based decision making.

\section{P050 'FOOD AND EATING BEHAVIOUR' - DEVELOPMENT OF A GUIDELINE FOR DUTCH PREVENTIVE CHILD HEALTH CARE}

C Lanting, N Heerdink-Obenhuiisen, M Kamphuis. Netherlands Institute for Applied Scientific Research, Leiden, Netherlands

\section{0:1136/bmjqs-2013-002293.140}

Background Many parents worry that their child is eating either too much or too little. In The Netherlands, Preventive Child Health Care (PCHC) is the main source of advice and information on food and eating behaviour. Yet, evidence on the subject is sparse and scattered. The Dutch Ministry of Health Welfare and Sports, therefore, requested a guideline; the PCHC-guideline should fit together with a guideline for paediatricians on the same subject, which was developed concurrently.

Objectives To develop a national guideline for PCHC-professionals on the subject to enhance uniform, evidence-based practice. Methods A working group of guideline developers, epidemiologists, and dieticians, together with professionals in PCHC, childpsychology and -pedagogics, and a pre-speech therapist developed the guideline, according to the principles of evidence-based medicine. Close collaboration took place with a group of paediatricians developing the new guideline 'Signalling somatic causes of abnormal nutritional behaviour in children'. Questions were formulated by the working group and evidence was extracted from literature, supplemented by practice-based consensus. The guideline was piloted in several PCHC settings.

Results Together with a group of child psychologists and psychiatrists, referral criteria for eating disorders were agreed on. Paediatricians formulated 'Somatic alarm symptoms'. Discussion Working with a large, interdisciplinary group of guideline 
developers carries the risk of collaboration problems, but when managed successfully, adds value to the end product.

Implications for Guideline Developers/Users In order to avoid collaboration problems, the scope of the guideline was agreed on in an early stage. Interdisciplinary collaboration enhanced guideline quality.

\section{P055 DOES THE CANADIAN TASK FORCE ON PREVENTIVE HEALTH CARE MEET THE INSTITUTE OF MEDICINE STANDARDS IN GUIDELINE DEVELOPMENT?}

${ }^{1} \mathrm{~S}$ Gorber, ${ }^{2} \mathrm{~A}$ Jaramillo, ${ }^{3} \mathrm{~K}$ Pottie, ${ }^{4} \mathrm{H}$ Singh. ${ }^{1}$ Public Health Agency of Canada, Ottawa, Canada; ${ }^{2}$ Public Health Agency of Canada, Ottawa, Canada; ${ }^{3}$ University of Ottawa, Ottawa, Canada; ${ }^{4}$ University of Manitoba, Winnipeg, Canada

\section{0:1136/bmjqs-2013-002293.141}

Background There are a plethora of guideline development groups in operation, each with its own methods for summarising evidence and processes for developing recommendations, which can leave consumers with questions about which guidelines to follow. In 2011 the Institute of Medicine (IOM) released a set of standards for developing trustworthy systematic reviews and clinical practice guidelines (CPGs). These standards address the structure, process, reporting and final products associated with evidence-based CPGs. The Canadian Task Force on Preventive Health Care (CTFPHC) was recently re-established with a mandate to develop clinical practice guidelines for primary care practitioners based on a systematic assessment of scientific evidence.

Objectives To compare the Canadian Task Force on Preventive Health Care's methods with the standards outlined in the IOM.

Methods The methods of the CTFPHC were compared to the IOM standards for both systematic reviews and CPGs and to the methods of other international guideline producers.

Results The CTFPHC methods are consistent with those of the IOM and international guideline development groups. Some differences include how patient and consumer input is incorporated into guidelines, the review of documents, and the final recommendation statements, the degree to which documents are publicly available and the processes for dissemination and knowledge translation.

Discussion New processes put in place to address the differences between CTFPHC methods and IOM standards will be explored.

Implications for Guideline Developers/Users Comparing the methods of guideline development groups to the IOM standards may provide users with a way to ascertain potential areas for enhancement of their CPG development process.

\section{P056 THE CANADIAN TASK FORCE ON PREVENTIVE HEALTH CARE: INTERPRETATION TOOL TO COMPARE PREVIOUS GRADING OF RECOMMENDATIONS TO GRADE}

L Dunfield, S Gorber, A Shane. Public Health Agency of Canada, Ottawa, Canada

10:1136/bmjqs-2013-002293.142

Background Guideline producers use various methods to grade their recommendations. The Canadian Task Force on Preventive Health Care (CTFPHC) previously assigned letter grades to their recommendations based on an evaluation of the evidence considering only study design. The CTFPHC now uses GRADE (Grading of Recommendations Assessment, Development and Evaluation), which considers the quality of the evidence and the strength of the recommendations.

Objectives The objectives were to develop a tool that would allow interpretation of the previous CTFPHC grading system to GRADE to enable comparison of guidelines.

Methods A comparison and mapping of each level of evidence and strength of recommendation for each grading system was undertaken. The methods working group and the knowledge translation working group of the CTFPHC reviewed the mapping system, which was then tested with a previous CTFPHC recommendation from 2001.

Results An interpretation tool which maps the level of evidence and the strength of recommendation was created and applied to a previous CTFPHC recommendation statement.

Implications for Guideline Developers/Users The tool is not meant to be a perfect system and does not require a formal assessment of the evidence from former guidelines. The evidence from previous guidelines is not assessed using GRADE, but rather interpreted for GRADE language. This tool will be useful in helping guideline developers compare guidelines using different grading systems and will allow the CTFPHC to compare previous guidelines to GRADE. This tool may also be applied when critically appraising guidelines from other guideline development groups.

\section{P058 DEVELOPING A YOUTH HEALTH CARE GUIDELINE ON SEXUAL DEVELOPMENT WITH LIMITED EVIDENCE}

${ }^{1} \mathrm{~S}$ Maris, ${ }^{2} \mathrm{~J}$ Deurloo, ${ }^{2} \mathrm{C}$ Lanting, ${ }^{2} \mathrm{M}$ Kamphuis, I van der Vlugt. ${ }^{1}$ Rutgers WPF, Utrecht, The Netherlands; ${ }^{2}$ TNO Child Health, Leiden, The Netherlands

\section{0:1136/bmjqs-2013-002293.143}

Background Children's sexual development starts at an early age and is a very complex subject, comprising the physical but also the psychosocial development. Youth Health Care (YHC) professionals can prevent and detect problems in sexual development, and play a guiding role in stimulating sexual competency and positive sexual attitudes.

Objectives Our goal was to develop an evidence based national YHC guideline for sexual development.

Methods The content of the guideline is based on (inter)national guidelines, literature searches, consensus and experience. The guideline is now piloted for use in daily practice by YHC professionals. The way we handled the limited amount of evidence and the results of this pilot will be presented. We cooperated with an international centre of expertise on sexual and reproductive health and performed literature searches for a selected number of questions.

Results The guideline describes the (physical and psychosocial) sexual development of children from 0-19 years old, determinants of sexual health and groups at risk. Discussion In this presentation, we would like to discuss the issue of dealing with the limited amount of evidence and we will show how we handled this issue. Working together with an experienced centre was crucial. Coming to consensus in the working group and performing a pilot test in addition, is essential in gaining obtaining support for the recommendations of the guideline.

Implications for Guideline Developers/Users In YHC not much evidence of high quality is available. Exchange of experiences will help other guideline developers dealing with this as well. 